\title{
Flow diversion for complex intracranial aneurysms in young children
}

\author{
Ramon Navarro, MD, ${ }^{1}$ Benjamin L. Brown, MD, ${ }^{1}$ Alexandra Beier, DO, ${ }^{2}$ Nathan Ranalli, MD, ${ }^{2}$ \\ Philipp Aldana, MD, ${ }^{2}$ and Ricardo A. Hanel, MD, $\mathrm{PhD}^{1-3}$
} ${ }^{1}$ Endovascular Surgical Neuroradiology, Mayo Clinic; ²Department of Pediatric Neurosurgery, University of Florida College of
Medicine; and ${ }^{3}$ Lyerly Neurosurgery, Baptist Hospital, Jacksonville, Florida

\begin{abstract}
Pediatric intracranial aneurysms are exceedingly rare and account for less than $5 \%$ of all intracranial aneurysms. Open surgery to treat such aneurysms has been shown to be more durable than endovascular techniques, and durability of treatment is particularly important in the pediatric population. Over the past 2 decades, however, a marked shift in aneurysm treatment from open surgery toward endovascular procedures has occurred for adults.

The authors describe their early experience in treating 3 unruptured pediatric brain aneurysms using the Pipeline embolization device (PED). The first patient, a girl with Majewski osteodysplastic primordial dwarfism Type II who was harboring multiple intracranial aneurysms, underwent two flow diversion procedures for a vertebrobasilar aneurysm and a supraclinoid internal carotid artery aneurysm. The second patient underwent PED placement on a previously coiled but enlarging posterior communicating artery aneurysm. All procedures were uneventful, with no postsurgical complications, and led to complete angiographic obliteration of the aneurysms. To the authors' knowledge, this is the first series of flow diversion procedures in children reported in the medical literature.

While flow diversion is a new and relatively untested technology in children, outcomes in adults have been promising. For challenging lesions in the pediatric population, flow diversion may have a valuable role as a well-tolerated, safe treatment with durable results. Many issues remain to be addressed, such as the durability of flow diverters over a very long followup and vessel response to growth in the presence of an endoluminal device.
\end{abstract}

http://thejns.org/doi/abs/10.3171/2014.9.PEDS14333

KEY WORDS pediatric; intracranial aneurysm; flow diversion; Pipeline embolization device; vascular disorders

$\mathrm{P}$ EDIATRIC intracranial aneurysms are exceedingly rare and account for less than 5\% of all intracranial aneurysms. The relative rarity of these lesions makes them difficult to manage and treat, but several other factors add to the clinical challenge. Pediatric aneurysms tend to present at a large size, have a propensity to grow, often occur in multiples, and, compared with their adult counterparts, they favor the posterior circulation. Open surgery to treat such aneurysms has been shown to be more durable than endovascular techniques, and durability of treatment is particularly important in the pediatric population. ${ }^{12}$ However, the factors listed above combined with the smaller anatomy and unique hemodynamic characteristics in the pediatric population can add considerable risk to open surgery and may offset the potential gains in durability.

Over the past 2 decades, a marked shift in aneurysm treatment from open surgery toward endovascular procedures has occurred for adults. More recently, this trend has been seen in the pediatric population..$^{10,15}$ In addition, endovascular coiling has been associated with fewer deaths and shorter hospital stays than those following clip placement. ${ }^{3}$ Given the constantly evolving neurointerventional tools and techniques, the range of intracranial aneurysms amenable to endovascular treatment has increased. Flow diverters represent one of the latest innovations in aneurysm treatment in adults, and to date, no recurrence has been reported after documented obliteration of an aneurysm

ABBREVIATIONS ACOA = anterior communicating artery; ICA = internal carotid artery; MCA = middle cerebral artery; $\mathrm{MOPD}=$ Majewski osteodysplastic primordial dwarfism; PCOA = posterior communicating artery; PED = Pipeline embolization device; $\mathrm{SAH}=$ subarachnoid hemorrhage; VB = vertebrobasilar.

SUBMITTED June 30, 2014. ACCEPTED September 15, 2014.

INCLUDE WHEN CITING Published online January 2, 2015; DOI: 10.3171/2014.9.PEDS14333.

DISCLOSURE The authors report no conflict of interest concerning the materials or methods used in this study or the findings specified in this paper. Dr. Hanel is a consultant for Covidien, Stryker, and Codman; has direct stock ownership in Codman and investment in Blockade; and has received grants from Microvention, outside of the submitted work. 
treated with flow diverters. While follow-up after flow diverter placement is relatively limited compared with the cumulative experience for clipping, the potential gain in treatment durability has promising implications for the pediatric population. In this paper, we present our early experience in the treatment of 3 pediatric brain aneurysms using the Pipeline embolization device (PED; ev3). To our knowledge, this is the first successful series of PED placements in children reported in the medical literature.

\section{Case Reports \\ Case 1}

History and Examination

An 11-year-old girl, $13 \mathrm{~kg}$ in weight, with a history of Majewski osteodysplastic primordial dwarfism Type II (MOPD Type II), was known to have multiple intracranial aneurysms after undergoing routine screening at 5 years of age for her underlying condition. An MR angiogram from that time showed a large anterior communicating artery (ACoA) aneurysm, a small basilar artery apex aneurysm, a right internal carotid artery (ICA) blister aneurysm, a right middle cerebral artery (MCA) bifurcation aneurysm, and bilateral posterior communicating artery (PCoA) aneurysms. Subsequently, when she was 6 years old, she underwent an elective left frontotemporal craniotomy for clipping of the ACoA and left PCoA aneurysms and wrapping of the left supraclinoid ICA at another institution. Four years after her initial procedure, she presented to the emergency department with severe headache, nausea, and vomiting, and head CT demonstrated subarachnoid hemorrhage (SAH).

In comparison with her previous imaging studies, the new CT scan revealed interval enlargement of the lateral and third ventricles, which raised concerns about the potential development of obstructive hydrocephalus. An external ventricular drain was placed, and a cerebral angiogram obtained at that time demonstrated multiple small intracranial aneurysms (right ICA anterior choroidal branch, right MCA anterior temporal branch, right ICA terminus, and left MCA). It also showed a small residual ACoA aneurysm despite its earlier clipping. Three larger lesions were seen at the basilar tip, vertebrobasilar (VB) junction, and left ICA (Fig. 1). Because of the blood pattern on CT and the aneurysm morphology, the basilar apex aneurysm was believed to be the source of bleeding, and the patient underwent balloon-assisted coil embolization. The hospital course was uneventful, and the patient was discharged home on Day 14.

\section{Treatment}

Approximately 3 months after her discharge, her parents were presented with the risks and benefits of observation versus microsurgical clipping versus endovascular treatment for the remaining larger lesions. For the unruptured aneurysms, a treatment plan was developed consisting of flow diverter placement from the right vertebral to basilar arteries together with aneurysm coiling and occlusion of the contralateral vertebral artery for the VB junction aneurysm and primary flow diverter placement for segmental disease of the left ICA. Dual antiplatelet thera- py consisting of $81 \mathrm{mg}$ of aspirin and $10 \mathrm{mg}$ of clopidogrel was administered daily, with platelet reactivity measured by the Accumetrics VerifyNow system to ensure therapeutic levels of aspirin and P2Y12 inhibition by clopidogrel before the procedure. The patient underwent two separate treatment sessions, the first for the VB junction aneurysm, involving placement of a PED $(2.5 \times 14 \mathrm{~mm})$ and coiling of the aneurysm sac and sacrifice of the contralateral vertebral artery without any complications. Three months later, she underwent a second procedure involving the placement of a PED for the left ICA dysplastic segmental disease, again without complication. At that time, the VB junction aneurysm was already completely obliterated.

\section{Posttreatment Course}

Follow-up imaging at 12 months after the last procedure showed obliteration of all the treated aneurysms (Fig. 1). Due to the technical difficulty of the initial craniotomy and the lack of good endovascular alternatives, the decision was made to observe several other small aneurysms.

\section{Case 2}

History and Examination

A 4-year-old boy, $14 \mathrm{~kg}$ in weight, with a previously coiled but enlarging right PCoA aneurysm, was found to have a recurrence of the aneurysm 11 months after a first endovascular procedure to treat it. This aneurysm was subsequently recoiled with near complete obliteration. On follow-up imaging 7 months after the second intervention, the boy had a new recurrence of the aneurysm.

\section{Treatment}

Microsurgery was offered at that time, but the family declined and instead opted to pursue another endovascular treatment. Off-label use of the PED was offered. The patient was given $81 \mathrm{mg}$ of aspirin and $15 \mathrm{mg}$ of clopidogrel for 7 days before the procedure. Therapeutic levels (as measured with Accumetrics VerifyNow) of the aspirin and clopidogrel were obtained. The boy underwent PED embolization without complication (Fig. 2).

\section{Posttreatment Course}

At the 3-month follow-up, MR angiography with gadolinium showed complete obliteration of the aneurysm, which was confirmed with a formal angiogram at the 6-month follow-up (Fig. 3).

\section{Discussion}

Pediatric brain aneurysms are uncommon and often complex. They tend to be large and fusiform, to occur in multiples, and to be located in the posterior circulation. These challenges are compounded in small children, in whom blood volume and hemodynamics play very important roles and whose small anatomy can increase the difficulty level of endovascular and open vascular approaches. Approximately $25 \%$ of pediatric intracranial aneurysms present in the 1st decade of life. ${ }^{6}$ It is estimated that large academic centers treat about 1 case per year. ${ }^{7}$ Thus, there is limited experience with this lesion, and most of the pub- 

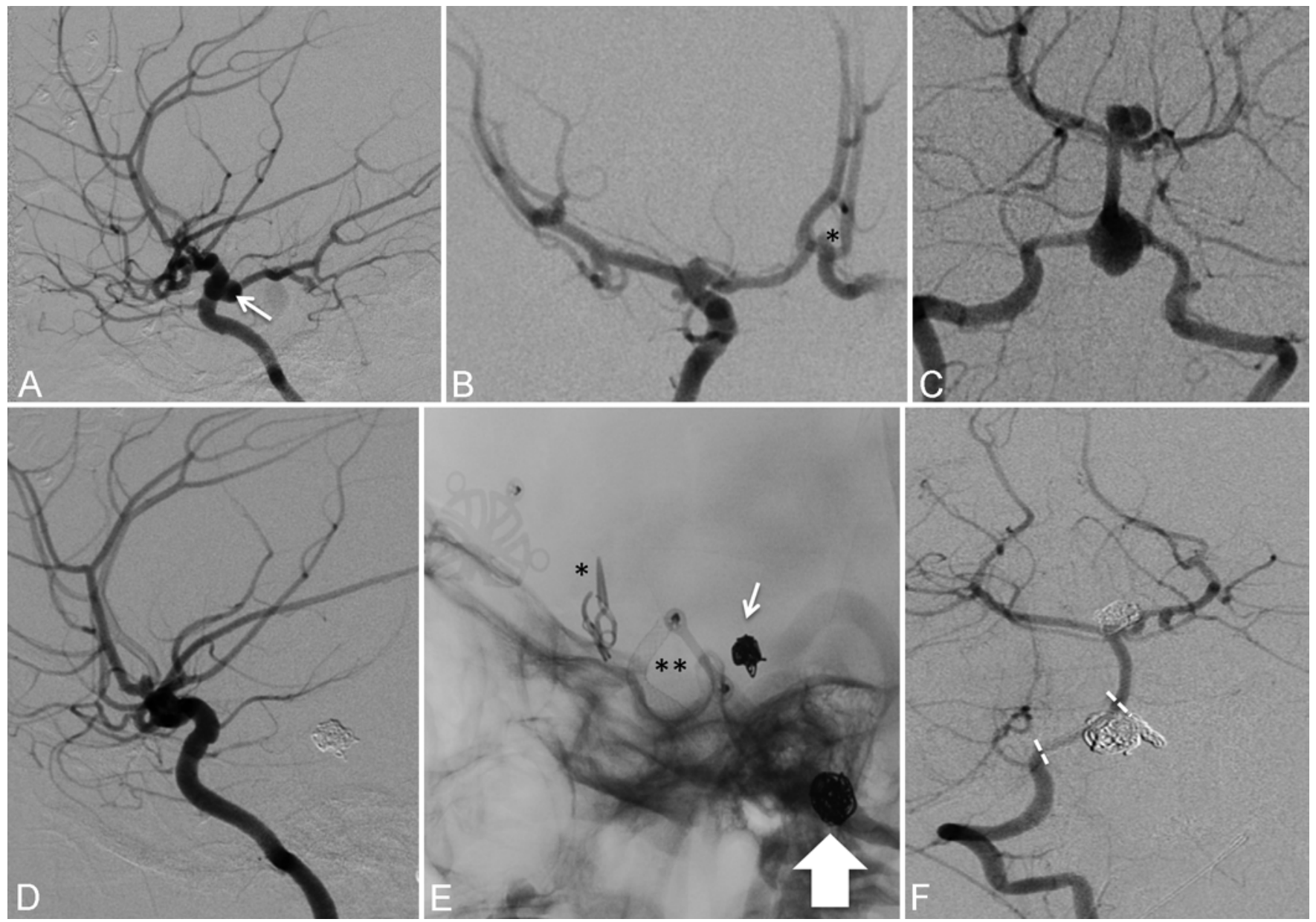

FIG. 1. Case 1. A: Right ICA injection, lateral view, showing a PCoA aneurysm (white arrow). B: Left ICA injection, anteroposterior view, showing aneurysms of the anterior choroidal artery, carotid terminus, and MCA bifurcation and a small residual ACOA aneurysm that had been previously coiled (asterisk). C: Right vertebral artery injection, anteroposterior view, shows a basilar tip and a VB aneurysm. D: Follow-up right PCoA angiogram, lateral view, shows good remodeling of the right ICA, no filling of the aneurysm, and decreased caliber of the PCoA. E: Lateral view of the skull depicting the complexity of treatment with combined surgery and endovascular approach to treat this child's multiple intracranial aneurysms. Two surgical clips are visible at the ACoA complex (asterisk), a PED is visible on the right ICA (double asterisks), and coils are visible at the VB junction and basilar tip (small arrow). The PED on the right vertebral artery next to the coil mass (large arrow) is not clearly visualized. F: Right vertebral artery injection angiogram showing good obliteration of the VB artery aneurysm by the PED with mild in-stent stenosis and occlusion of the basilar tip aneurysm. White dashed lines show approximate extension of the flow diverter.

lished series are small, ${ }^{6,712-14}$ which leads to many extrapolations from the adult literature. This may be problematic given that pediatric aneurysm and patient characteristics are generally very different from their adult counterparts. Up to $30 \%$ of pediatric patients with intracranial aneurysms have medical comorbidities, such as connective tissue disorders, polycystic kidney disease, traumatic aneurysms, and infectious aneurysms, among others, as well as a history of irradiation. ${ }^{9,10,14}$ The patient in Case 1 had MOPD Type II. This is a rare autosomal recessive disorder that presents with intrauterine growth retardation, short stature of approximately $100 \mathrm{~cm}$ at adulthood, characteristic facial features, skeletal dysplasia, and microcephaly. Up to $52 \%$ of this population has neurovascular abnormalities, namely intracranial aneurysms and moyamoya syndrome. ${ }^{5}$ Patients with this disease are advised to undergo neuroimaging surveillance once a year. Intracranial surgery in children with MOPD Type II could present problems. The patients and brain vasculature are small, surgical fields are diminutive, and arteries appear abnormally tortuous and friable (G.K. Steinberg, personal communication, 2014). ${ }^{16}$ The patient in Case 1 also had segmental disease of the cavernous and supraclinoid carotid arteries with multiple aneurysms and a complex VB junction aneurysm. We thought that a PED would treat these multiple lesions while avoiding major craniotomies and multiple coiling procedures. A direct approach for clipping the ACoA aneurysm had been previously performed (4 years prior to the SAH caused by the basilar tip aneurysm rupture), deemed very complex, with an intraoperative aneurysm rupture that was difficult to control.

A patient's expected lifespan also plays a critical role when deciding on the best type of therapy. Treatment must be efficacious and durable since most patients have decades of life ahead. As in adults, endovascular treatment is becoming more and more the treatment of choice for 

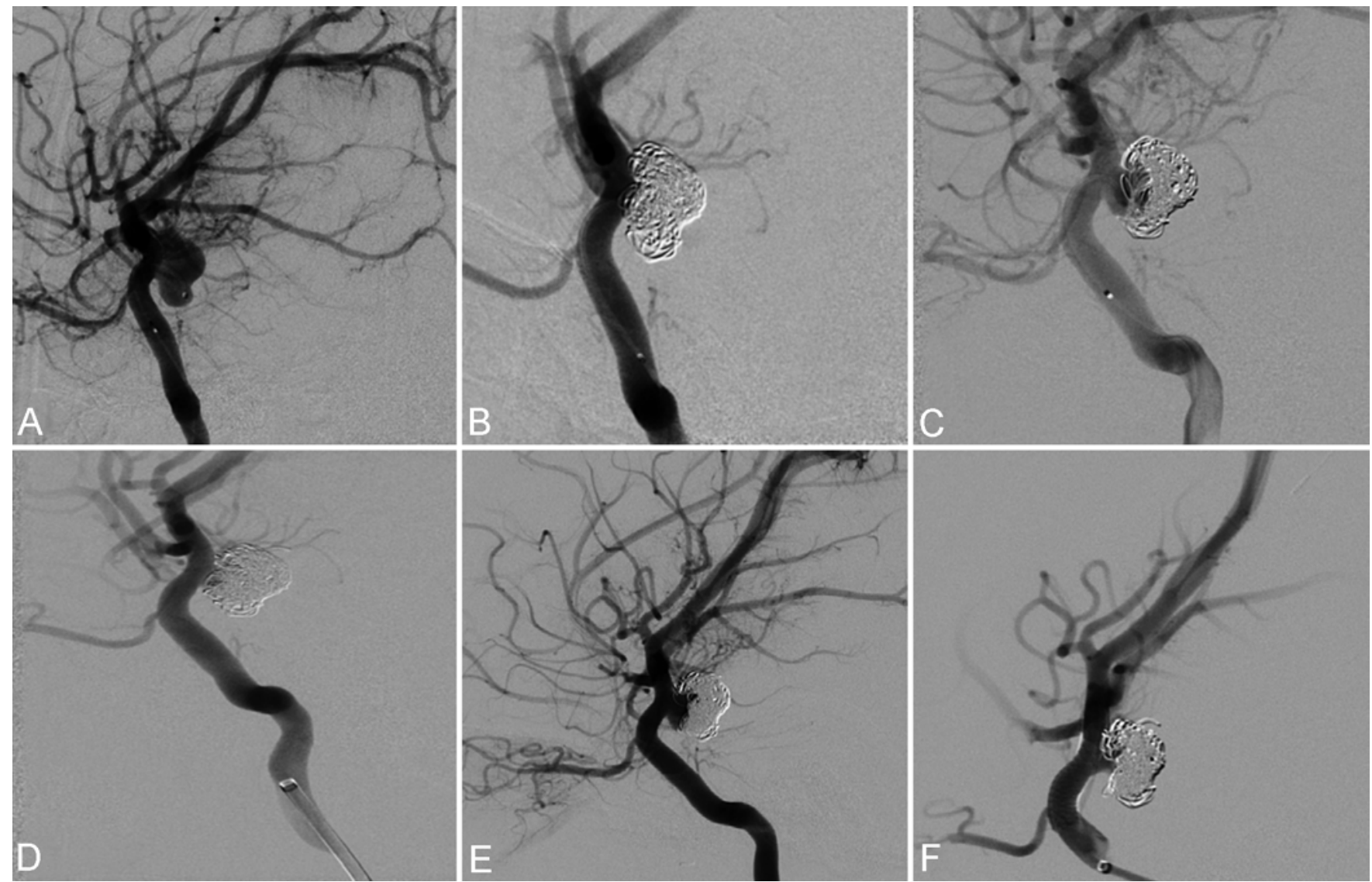

FIG. 2. Case 2. Initial angiographic presentation of the right $P C O A$ aneurysm $(A)$ and the angiographic result after the first treatment (B). First recurrence of the aneurysm (C) and the angiographic result after the second primary coiling procedure, showing a small residual neck (D). Second recurrence due to coil compaction (E) and final lateral view (F) after placement of a PED acrosS the aneurysm neck.

intracranial aneurysms in children. ${ }^{10,15}$ Moreover, Alawi et al. have recently reported higher mortality in children treated with surgical clipping (OR 2.50) than in those who underwent endovascular treatment. ${ }^{3}$ Nevertheless, one of the drawbacks of the neurointerventional technique is that
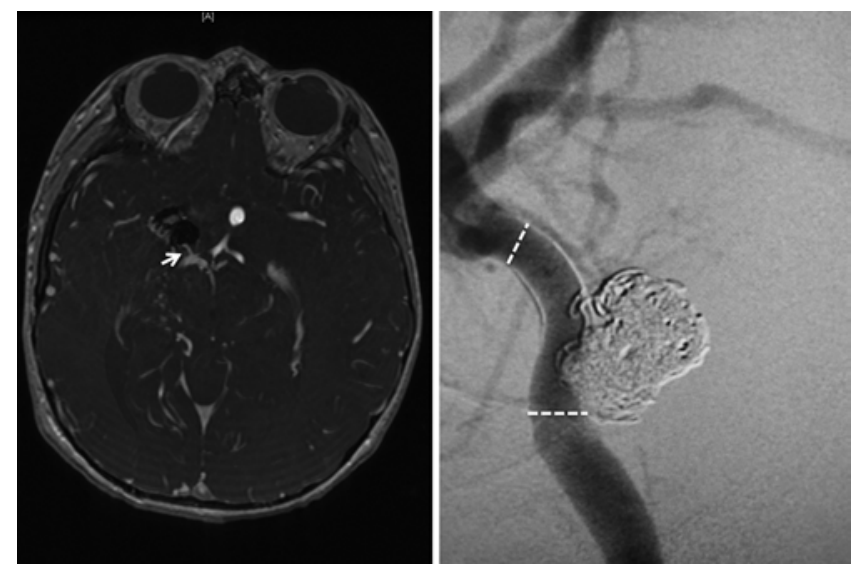

FIG. 3. Case 2. Left: Axial MR angiogram obtained 3 months after placement of the flow diverter. No filling of the aneurysm is visible, with patency of the right PCoA (white arrow) and right ICA. Right: This result was confirmed with a formal digital subtraction angiogram 6 months after PED placement. White dashed lines indicate extension of the PED. it may not be permanent. Sanai et al. reported a $14 \%$ rate of recurrence in their series of pediatric intracranial aneurysms treated endovascularly compared with a $0 \%$ recurrence rate in children treated with open surgery. ${ }^{12}$ Other groups also advocate surgical therapy as a first option for aneurysms that present with rupture. ${ }^{14}$ Two reasonably large series have reported $15 \%-23 \%$ perioperative complications for open surgical treatment, along with a $94 \%$ obliteration rate and a $2.6 \%$ recurrence rate per year. ${ }^{8,12}$ However, as many authors anticipated, ${ }^{7,10,12}$ instruments and endovascular techniques are rapidly evolving and replacing open surgery as the treatment of choice in many cases. Recent series have shown better clinical results with endovascular treatments than with open surgery ${ }^{2}$ and an obliteration rate of $96 \%$ with a mean 3-year follow-up. ${ }^{13}$

Flow diversion is a relatively new technique that is increasingly used to treat intracranial aneurysms. In the United States, the most widely used flow diverter is the PED. It is a self-expanding bimetallic stent-like device that has a higher metal surface coverage (approximately 30\%-35\%) and lower porosity than other stents. The PED helps to divert flow away from the aneurysm, and thus promoting early thrombosis. The dense scaffold then facilitates neointimal growth with parent vessel remodeling. A recent review of the PED literature in adults, which included 905 patients with 1043 aneurysms, showed an 80\% occlusion rate at 6 
months with a $2.3 \%$ mortality rate and a $16.7 \%$ cumulative event rate. ${ }^{11}$ Nearly $50 \%$ of these cases were large or giant aneurysms that would represent challenging open surgery cases. More importantly, there has not been a single report in the literature of recanalization once obliteration of an aneurysm has occurred. The PED is a major evolution in the endovascular field, and its indications for use will probably continue to expand. To our knowledge, only one case of a young child (10 years old) treated successfully with a flow diverter has been published in the medical literature. ${ }^{4}$ Neurosurgeons in that case used a SILK flow diverter (Balt Extrusion) composed of 48 braided nitinol strands to treat a 22-mm, infectious cavernous sinus aneurysm, with complete occlusion at the 3-month follow-up. There are also reports of telescoping stents used in a "flow diverter" fashion as well as flow reversal to treat aneurysms in this population..$^{13}$ In addition, Abla and colleagues described failed treatment with multiple PEDs in different stages for a giant supraclinoid aneurysm in a 10 -year-old child. ${ }^{1}$ Their patient had to undergo open surgical bailout with parent vessel occlusion and extracranial-intracranial bypass to alleviate rapid, progressive visual loss due to enlarging intraaneurysmal thrombus.

At our center, both open and endovascular techniques are available on a daily basis, and the ultimate choice of a treatment modality is decided by a team of pediatric neurosurgeons and dual-trained cerebrovascular neurosurgeons in consultation with the patient's parents. In Case 1, our first contact with the patient was for the treatment of an acutely ruptured basilar artery aneurysm. At that time, we decided not to address the remaining aneurysms until she had recovered from the SAH. To place flow diverters, the patient must be on dual antiplatelet agents to avoid thromboembolic complications given that the flow diverters have a high metal surface coverage. For this reason, among others, flow diverters are not well suited for acutely ruptured aneurysms. Patients are started on $81 \mathrm{mg}$ of aspirin and $0.2-1 \mathrm{mg} / \mathrm{kg} /$ day of clopidogrel 1 week before the procedure. We routinely measure platelet reactivity to aspirin and clopidogrel using the Accumetrics VerifyNow test because there is significant platelet response variability among the population. Antiplatelet dose is titrated to obtain therapeutic levels (aspirin reactivity units below 550 and P2Y12 reactivity units between 20 and 194). Although this issue is controversial, we believe that achieving therapeutic levels of anti-aggregation is important to avoid postoperative ischemic complications. After surgery, clopidogrel administration is maintained for at least 6 months and baby aspirin is maintained for life. In Case 2, a 4-year-old child had a second recurrence of a previously coiled, probably dissecting PCoA aneurysm. Surgery was the first-line treatment recommended at that time, but it was refused by the family. We hypothesized that the fast-growing vasculature of the child during the maximum brain growth period (birth to 4 years) and/or dissection as the cause of the aneurysm might have hindered epithelialization of the neck of the aneurysm, which also might have been growing in size and thus endangering the durability of a coil construct.

There are potential limitations in using flow diverters to treat intracranial aneurysms. If this treatment strategy fails, access to the aneurysm for further coiling cannot be achieved because of the low porosity of the devices. Therefore, adding another device or open surgery is the remaining therapeutic option. Further, covered side branches of the parent vessel and/or aneurysm can be angiographically lost because of flow reduction. This tends to be asymptomatic; however, the fate of these vessels is unknown since long-term follow-up is lacking. In addition, in-stent stenosis greater than $50 \%$ has been documented in up to $10 \%$ of cases. ${ }^{11}$ This effect is also likely to remain clinically silent. In the cases we present, the PED length and diameter were tailored to the parent vessel size. The 3 pediatric aneurysms described herein had no clinical complications with complete aneurysm occlusion. Long-term follow-up imaging in cases like ours will provide some additional information in this respect. Regardless, serial long-term follow-up imaging is mandatory in these children since de novo formation of an aneurysm is a recognized phenomenon with an estimated incidence of $8 \%-12 \% .{ }^{8,12}$

\section{Conclusions}

While flow diversion is a new and relatively untested technology in children, its results in adults have been promising. For challenging lesions in the pediatric population, flow diversion may have a valuable role as a welltolerated, safe treatment with durable results. Many issues remain to be addressed, such as the durability of flow diverters over the long term and vessel response to growth in the presence of an endoluminal device.

\section{References}

1. Abla AA, Zaidi HA, Crowley RW, Britz GW, McDougall $\mathrm{CG}$, Albuquerque FC, et al: Optic chiasm compression from mass effect and thrombus formation following unsuccessful treatment of a giant supraclinoid ICA aneurysm with the Pipeline device: open surgical bailout with STA-MCA bypass and parent vessel occlusion. J Neurosurg Pediatr 14:31-37, 2014

2. Agid R, Souza MP, Reintamm G, Armstrong D, Dirks P, TerBrugge KG: The role of endovascular treatment for pediatric aneurysms. Childs Nerv Syst 21:1030-1036, 2005

3. Alawi A, Edgell RC, Elbabaa SK, Callison RC, Khalili YA, Allam H, et al: Treatment of cerebral aneurysms in children: analysis of the Kids' Inpatient Database. J Neurosurg Pediatr 14:23-30, 2014

4. Appelboom G, Kadri K, Hassan F, Leclerc X: Infectious aneurysm of the cavernous carotid artery in a child treated with a new-generation of flow-diverting stent graft: case report. Neurosurgery 66:E623-E624, 2010

5. Bober MB, Khan N, Kaplan J, Lewis K, Feinstein JA, Scott CI Jr, et al: Majewski osteodysplastic primordial dwarfism type II (MOPD II): expanding the vascular phenotype. Am J Med Genet A 152A:960-965, 2010

6. Garg K, Singh PK, Sharma BS, Chandra PS, Suri A, Singh M, et al: Pediatric intracranial aneurysms-our experience and review of literature. Childs Nerv Syst 30:873-883, 2014

7. Huang J, McGirt MJ, Gailloud P, Tamargo RJ: Intracranial aneurysms in the pediatric population: case series and literature review. Surg Neurol 63:424-433, 2005

8. Kakarla UK, Beres EJ, Ponce FA, Chang SW, Deshmukh VR, Bambakidis NC, et al: Microsurgical treatment of pediatric intracranial aneurysms: long-term angiographic and clinical outcomes. Neurosurgery 67:237-250, 2010 
9. Lasjaunias PL, Campi A, Rodesch G, Alvarez H, Kanaan I, Taylor W: Aneurysmal disease in children. Review of 20 cases with intracranial arterial localisations. Interv Neuroradiol 3:215-229, 1997

10. Liang J, Bao Y, Zhang H, Wrede KH, Zhi X, Li M, et al: The clinical features and treatment of pediatric intracranial aneurysm. Childs Nerv Syst 25:317-324, 2009

11. Murthy SB, Shah S, Venkatasubba Rao CP, Bershad EM, Suarez JI: Treatment of unruptured intracranial aneurysms with the pipeline embolization device. J Clin Neurosci 21:6-11, 2014

12. Sanai N, Quinones-Hinojosa A, Gupta NM, Perry V, Sun PP, Wilson CB, et al: Pediatric intracranial aneurysms: durability of treatment following microsurgical and endovascular management. J Neurosurg 104 (2 Suppl):82-89, 2006

13. Saraf R, Shrivastava M, Siddhartha W, Limaye U: Intracranial pediatric aneurysms: endovascular treatment and its outcome. J Neurosurg Pediatr 10:230-240, 2012

14. Stiefel MF, Heuer GG, Basil AK, Weigele JB, Sutton LN, Hurst RW, et al: Endovascular and surgical treatment of ruptured cerebral aneurysms in pediatric patients. Neurosurgery 63:859-866, 2008

15. Vasan R, Patel J, Sweeney JM, Carpenter AM, Downes K, Youssef AS, et al: Pediatric intracranial aneurysms: current national trends in patient management and treatment. Childs Nerv Syst 29:451-456, 2013

16. Waldron JS, Hetts SW, Armstrong-Wells J, Dowd CF, Fullerton HJ, Gupta N, et al: Multiple intracranial aneurysms and moyamoya disease associated with microcephalic osteodysplastic primordial dwarfism type II: surgical considerations. J Neurosurg Pediatr 4:439-444, 2009

\section{Author Contributions}

Conception and design: Navarro, Hanel. Acquisition of data: Beier. Analysis and interpretation of data: Navarro. Drafting the article: Navarro, Brown. Critically revising the article: Beier, Ranalli, Aldana, Hanel. Reviewed submitted version of manuscript: all authors. Approved the final version of the manuscript on behalf of all authors: Navarro. Curated images: Hanel. Creation of images: Navarro.

\section{Correspondence}

Ramon Navarro, Neurological Institute, Cleveland Clinic Abu Dhabi, United Arab Emirates. email: navarrr2@cleveland clinicabudhabi.ae. 